\title{
ENHANCING UNDERWATER IMAGES USING PIECEWISE LINEAR SMOOTHING GRADIENT GUIDED FILTER
}

A. Chrispin Jiji

Assistant Professor, Department of Electronics and Communication Engineering, The Oxford College of Engineering, Bangalore-560068 and affiliated to Visvesvaraya Technological University, Belagavi, Karnataka, (India). E-mail: chrispinjij@gmail.com ORCID: https://orcid.org/0000-0001-5267-788X

N. Ramrao

Vice Chancellor, Kalasalingam University, Srivilliputtur, Tamilnadu, (India). E-mail: nagaraj.ramrao@gmail.com ORCID: https:// orcid.org/0000-0003-2542-5999

\section{Citación sugerida:}

Chrispin Jiji, A., y Ramrao, N. (2020). Enhancing underwater images using piecewise linear smoothing gradient guided filter. 3C Tecnología. Glosas de innovación aplicadas a la pyme. Edición Especial, Marzo 2020, 129-139. http://doi.org/10.17993/3ctecno.2020.specialissue4.129-139

\section{Suggested citation:}

Chrispin Jiji, A., \& Ramrao, N. (2020). Enhancing underwater images using piecewise linear smoothing gradient guided filter. 3C Tecnología. Glosas de innovación aplicadas a la pyme. Edición Especial, Marzo 2020, 129-139. http://doi.org/10.17993/3ctecno.2020.specialissue4.129-139 


\section{ABSTRACT}

Poor visibility owing towards illumination absorption and scattering is challenging for processing undersea descriptions. However, for enhancing true scene from such degradation is more important. Unfortunately, existing methods cause gradient reversal artifact particularly near boundaries. To get better insight of undersea imagery, we project a piecewise linear smoothing Gradient Guided Filter (P-GGF) technique is to defeat the difficulties caused by conventional schemes, hence produce sharper boundaries based on GGF and smoothed output based on piecewise linear model. The projected technique mainly functional for smoothing, flash and feathering. Tentative results prove that the resultant algorithm can produce imagery with improved ocular excellence than existing methods.

\section{KEYWORDS}

Image Enhancement, Guided Filter, Piecewise liner smoothing, Piecewise constant smoothing. 


\section{INTRODUCTION}

Discovering an unexplained undersea globe has paying attention in modern era. Clear descriptions in oceanic surroundings take part a significant task in discovering as well as inspecting underneath globe, namely to observe oceanic biodiversity, undersea salvage, perceiving submerged tube drip, undersea computer visualization applications etc. Here the submerged imaging finds a large-scale application. Some difficulty comes all throughout underneath descriptions of illumination absorption as well as scattering. Deep-rooted design of ocean also composes complications in undersea. On the design of the ocean, reflection of the illumination alters. The reflected radiance is horizontally polarized along with its halfway gets inside the water vertically. Vertical polarization has significant property that hatches the substance not as great shining and aides on the way to capture deep colors. One more difficulty in underneath descriptions associated towards underneath density as 800 bits impenetrable than air. Hence when beam travels from air into water, it is halfway back and, in the meantime, partially enters the water. As we go deeper into ocean, dimension of beam underneath starts reducing. The underneath molecules absorb assertive size of beam and create problem for capturing imagery. That is the reason; undersea descriptions are getting darker as depth increases. The color with shorter wavelength travels remoteness as compared to longer wavelength. This is why undersea descriptions conquered only with blue color as in Torres-Méndez and Dudek (2005), Chiang and Chen (2012).

There are many challenges that enhance visibility of corrupted descriptions. While weakening of submerged descriptions outcomes the combination of multiplicative as well as additive procedures in Schettini and Corchs (2010) conventional improvement system namely contrasts alteration, histogram equalizer is robustly defective for such assignment. Former mechanism to review in section II, difficulty was attempted with customized attainment strategy by several imagery in Narasimhan and Nayar (2003), specific module in He and Seet (2004) or divergence methods in Schechner and Averbuch (2007). Regardless of their accomplishment, above approaches undergo various problems which degrade the system performance.

In contrast, this paper proposes new method for enhancing undersea descriptions using Piecewise linear smoothing. Our approach uses Piecewise linear smoothing gradient 
guided filter for getting better picture. Numerous spatial field schemes use bilateral scheme, which causes blurring and gradient deformation. Gradient Guided representation performs filtering process using guidance picture substance. Thus, a boundary preservation method mainly to improve excellence of underneath descriptions.

This paper prepared as follows. Foremost, in Section 2, we concise the existing schemes. Section 3 introduces a comparison of conventional and projected filters. Section 4 describes a new method for enhancing undersea descriptions. In Section 5, we describe tentative outcomes and at last Section 6 conclude our method.

\section{RELATED WORK}

Edge-preserving smoothing is the fundamental processing procedure within several lowlevel computer visualization applications in Farban, Fattal and Lischinski (2010), Farbman, Fattal, Lischinski, and Szeliski (2008), Gastal and Oliveira (2011; 2012). Meant for on whole smooth filters believe the smoothed output imagery are piecewise constant. Generally, the edge-preserving techniques using confined filtering to keep sharp boundaries. Bilateral filter is extensively used because of its effortlessness Tomasi and Manduchi (1998). Conversely, it undergoes unwanted sharpening of edges may show undesired profiles around edges. Guided filter introduced in He, Sun, and Tang (2013) overcome these problems but show unwanted smoothing edges. Weighted GF in Li et al. (2015) uses gradient-domain constrains for smoothing the picture elements but in few cases, it cannot preserve the boundaries. The gradient domain GIF in Kou, Chen, Wen, and $\operatorname{Li}$ (2015) incorporates a precise initial-order boundary-aware restraint to keep up boundaries better in some cases.

These conventional schemes are typically denoted as local model which causes artifact such as gradient reversals, hence may not fine for few cases. For those schemes, a piecewise linear form preferred mostly for properly smooth out boundaries. So, no artifacts are present in improved results. In Liu et al. (2018) piecewise linear method via guided representation accurately resolve difficulty of gradient-reversal except that only some cases illustrate small smoothing boundaries.

Therefore, we project a P-GGF to properly sharp, smooth all boundaries as well as do artifacts free enhanced result. Three major goals of projected sections as follows: 
1. To take away gradient reversal, we use Gradient Guided Filter (GGF) to give sharper boundaries.

2. Next, we use piecewise linear smoothing to smooth the boundaries.

3. The projected module uses P-GGF to produce improved output.

Experimental results produced by P-GGF can perfectly remove the problems caused by the conventional method.

\section{PIECEWISE LINEAR SMOOTHING}

Conventional methods mostly suitable for image regions more likely be piecewise linear may cause artifacts. Clearly, detail layers cannot correctly say details in the original descriptions. Thus, gradient reversal artifacts exist in their enhanced imagery. In highlighted regions, two kinds of smoothing can properly keep strong gradients which means strong edges are properly preserved. For weak edges of small gradients which should be smoothed out. However, these gradients are not properly smoothed by conventional methods or even improperly sharpened. As a result, gradient reversal artifacts exist with the enhanced representation.

For most cases, conventional methods usually need a huge number of bins to avoid quantization artifacts. Smoothed and enhanced descriptions achieved with first smoothing of conventional filters and later uses smooth gradient for reconstructing the image. The reason for these phenomena is clear. The classical smoothing performed in intensity domain where intensity values could be very large. In contrast, to overcome all problems we go for proposed method namely piecewise linear method.

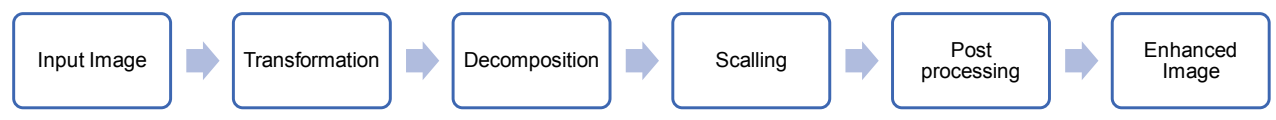

Figure 1. Block diagram of Proposed Method. 


\section{PROPOSED METHOD}

As depicted in Figure 1 our technique is mainly for enhancing underwater images using piecewise linear smoothing. Initially, original picture developed by RGB or HSV color space. RGB depict colors in combination of primary colors.

HSV describes colors namely Hue, Saturation, and Value. Color depiction acting an essential task, HSV form often chosen over RGB form and express color likewise to how human vision lean-to recognize color. Later, we used first layer decomposition by smoothing the image using L1L0 smoothing and second layer decomposition for sharpening the edges using gradient guided filter. Next, scaling to vary ocular form of a picture. Finally, post processing of scaled image to enhance the quality of the image.

The conventional method can simply modeled as:

$$
E_{P}^{\wedge}=a_{k}, P \in N(k)
$$

where $E_{P}^{\wedge}$ represents expected output pixel rate at location $P=\left[P_{x}, P_{y}\right], a_{k}$ denotes expected constant rate of pixel values inside kth area in the picture indicated as $N(k)$. In contrast to conventional model assumption, projected method can signify:

$$
E_{P}^{\wedge}=a_{k} G_{P}+b_{k}, P \in w(k)
$$

where $E_{P}^{\wedge}$ denotes expected output pixel value at position $\mathrm{p}, G_{P}$ represents pixel value of guidance image at position p. $a_{k}$ and $b_{k}$ stay steady in $w(k)$. These methods show no gradient reversal artifacts.

In this paper, we assume a linear form which is spatially linear different from gradient guided image filter. In addition, our method also focuses on how to do projected method using classical methods.

Different from piecewise linear function, we formulate imagery as:

$$
E_{P}^{\wedge}=a_{k}^{T} P+b_{k}, P \in N(k)
$$


where $a_{k}=\left[a_{k}^{x}, a_{k}^{y}\right]^{T}$ as well as $b_{k}$ denotes some linear coefficients which assumed to stay constant inside $N(k)$. The above equation indicates linear polynomial regression to remove stair-casing effect. The model in (3) is general and abstract form which is not used for explicit formulations of filters. However, when we take the derivative of $E_{P}^{\wedge}$ with respect to $\mathrm{p}$, then we have:

$$
\frac{\partial E_{P}^{\wedge}}{\partial P}=a_{k}, P \in N(k)
$$

Note that $\frac{\partial E_{P}^{\wedge}}{\partial P}$ signifies gradient of picture at p. For linear form, their gradients are piecewise constant. Though, dissimilarity assumes resultant imagery of smoothed output gradients. Conversely, problem is that we cannot simply reconstruct picture only by its smoothed gradients. The difficulty in using original and its filtered gradients is to reconstruct filtered image. For original picture, its x-axes and y-axes gradients denoted as $\partial x I_{0}$ and $\partial y I_{0}$. By denoting smoothing process of piecewise constant filters as $\mathfrak{\Im}_{p c}($.$) , then final representation$ $E$ reconstructed by minimizing the following energy function:

$$
\left\|\hat{E}-E_{0}\right\|^{2}+\beta\left\{\left\|\partial x \hat{E}-\mathfrak{J}_{p c}\left(\partial x E_{0}\right)\right\|^{2}-\left\|\partial y \hat{E}-\mathfrak{J}_{p c}\left(\partial y E_{0}\right)\right\|^{2}\right\}
$$

From this, we can execute piecewise linear smoothing through conventional scheme in the following two steps:

(1). Smoothing x-axes in addition to y-axes, gradients $\partial x E_{0}$ and $\partial y E_{0}$ of original representation $E_{0}$ with $\mathfrak{I}_{p c}($.$) . The smoothed output gradients are denoted by \mathfrak{I}_{p c}\left(\partial x E_{0}\right)$ and $\mathfrak{I}_{p c}\left(\partial y E_{0}\right)$

(2). Using (5) for enhancing picture $\hat{E}$ from $E_{0}, \mathfrak{I}_{p c}\left(\partial x E_{0}\right)$ and $\mathfrak{I}_{p c}\left(\partial y E_{0}\right)$ with a proper value of $\beta$. Then the enhanced $\hat{E}$ is spatially piecewise linear as modelled in (3).

\section{EXPERIMENTAL RESULTS}

This section presents a comparison of improved outcome produced via ours and conventional schemes through subjective evaluation of test scene. Figure 2 gives improved result than conventional methods. As seen from Figure 2(a)-(f), all schemes get better output to some 
extent. Figure 2(a) bilateral process Tomasi and Manduchi (1998) results enhanced scene, but it cannot keep sharp boundaries. In Figure 2(b) He et al. (2013) discussed Guided filter to keep boundaries sharper but fail to represent the picture well close to a few boundaries. To defeat Li, Zheng, Zhu, Yao and Wu (2015) used weighted guided scheme in Figure 2(c) used for minimizing halo artifacts but fail to keep the boundaries.

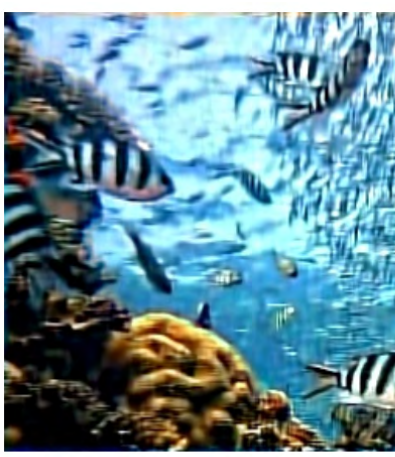

a) (Tomasi and Manduchi, 1998)

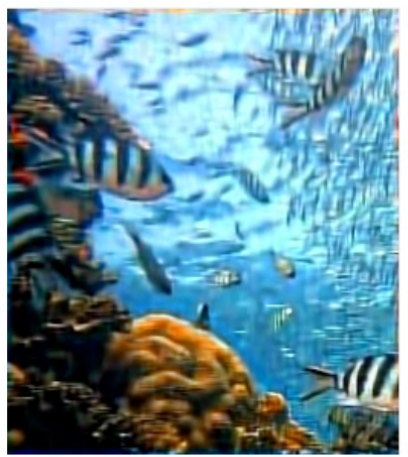

d) (Kou et al., 2015)

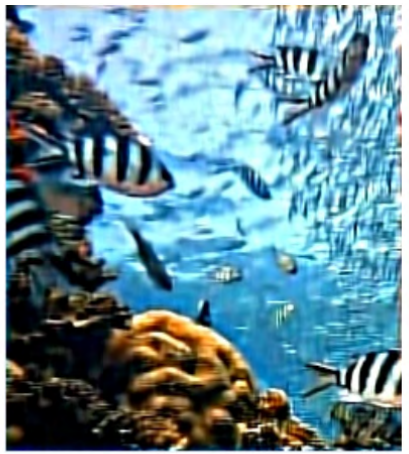

b) (He et al., 2013)

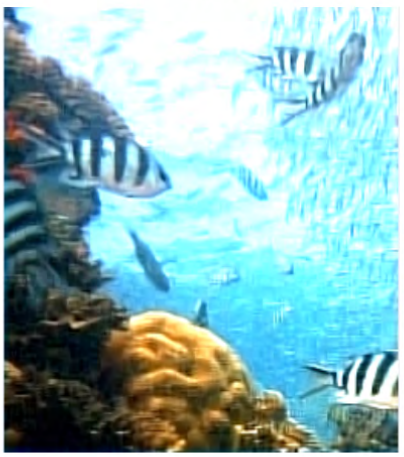

e) Liu et al. (2018)

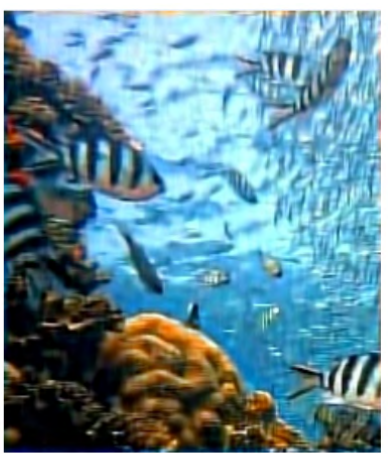

c) (Li et al., 2015)

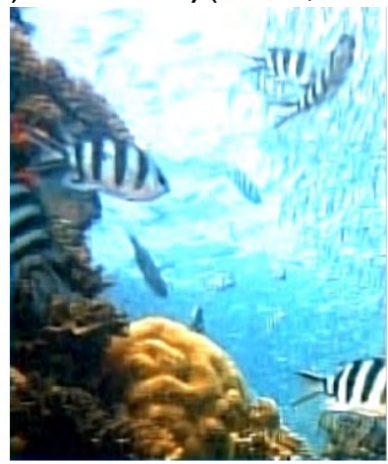

f) Own elaboration

Figure 2. Enhancing performance evaluation of Underwater Imagery.

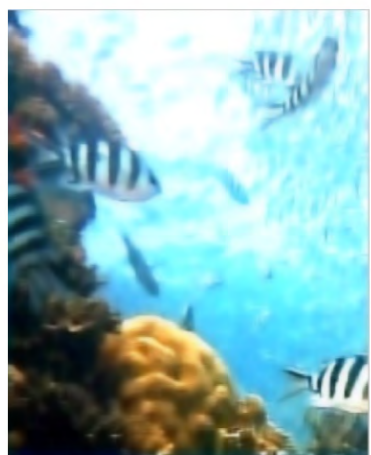

a)

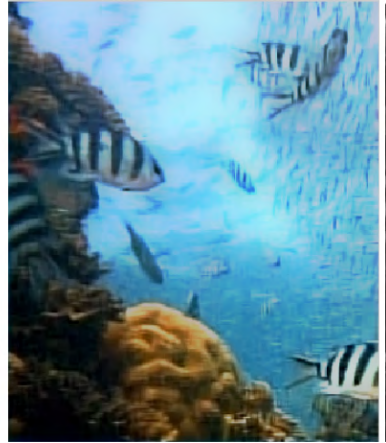

b)

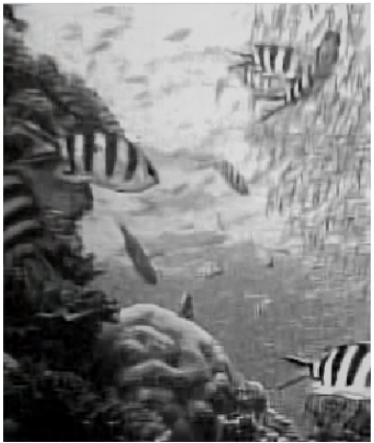

c)

Figure 3. Various applications of Proposed Method a) Smoothing b) Flash c) Feathering. 
Figure 2(d), Kou et al. (2015) used an edge aware factor introduced to keep up well-built boundaries but smoothed away weak boundaries. Piecewise linear method via guided representation Liu et al. (2018) in Figure 2(e) accurately resolve difficulty of gradient-reversal except that only some cases illustrate small smoothing boundaries. At last projected approach Figure 2(f) outcomes strong and weak boundaries accurately and makes the standardized system into range $[0 ; 1]$ earlier to smoothing and then standardize back towards original range after smoothing. Figure 3 gives various applications of projected scheme to smooth out weak boundaries, drop artifacts using flash filtering and refine boundaries using feathering.

However, the projected scheme gives improved results than the schemes in Tomasi and Manduchi (1998), He et al. (2013), (Li et al., 2015), Kou et al. (2015), Liu et al. (2018). Therefore, projected scheme is more suitable for various applications.

\section{CONCLUSIONS}

We projected a piecewise linear smoothing Gradient Guided Filter (P-GGF) method to improve undersea descriptions. The proposed method properly handles gradient reversal artifacts caused by conventional and uses GGF with sharper boundaries and smoothed result based on piecewise linear model. Overall, projected scheme P-GGF can effectively improve the scene. Experimental results prove that projected method generate imagery by improved ocular excellence than conventional methods. We believe that proposed technique used for many applications such as smoothing, Flash and feathering. The extra remarkable difficulty based on the expansion of the projected method to obtain fine details from numerous descriptions concurrently using extensive filter in Li et al (2012; 2014). We leave this for future research.

\section{ACKNOWLEDMENT}

We gratefully thank the Visvesvaraya Technological University, Jnana Sangama, Belagavi for financial support extended to this Research work. 


\section{REFERENCES}

Ghiang, J. Y., \& Chen, Y. G. (2012). Underwater image enhancement by wavelength compensation and dehazing. IEEE Transactions on Image Processing, 31(4), 1756-1769. https:/ / doi.org/10.1109/TIP.2011.2179666

Farban, Z., Fattal, R., \& Lischinski, D. (2010). Diffusion maps for edge-aware image editing. ACM Transa Grap. https://www.cse.huji.ac.il/labs/cglab/projects/diffmaps/ diffmaps.pdf

Farbman, Z., Fattal, R., Lischinski, D., \& Szeliski, R. (2008). Edge-preserving decompositions for multi-scale tone and detail manipulation. ACM Transactions on Graphics, 27(3). https://doi.org/10.1145/1360612.1360666

Gastal, E. S. L., \& Oliveira, M. M. (2011). Domain transforms for edge awareimage and video processing. ACM Transactions on Graphics, 30(4). https://doi. org/10.1145/2010324.1964964

Gastal,E.S.L., \&Oliveira,M.M.(2012).Adaptivemanifoldsforreal-timehigh-dimensional filtering. ACM Transactions on Graphics, 31(4). https://doi.org/10.1145/2185520.2185529

He, D. M., \& Seet, G. G. L. (2004). Divergent-beam LiDAR imaging in turbid water. Optics and Lasers in Engineering, 41(1), 217-231. https://doi.org/10.1016/S0143-8166(02)00138-0

He, K., Sun, J., \& Tang, X. (2013). Guided image filtering. IEEE Transactions on Pattern Analysis and Machine Intelligence, 35(6), 1397-1409. https://doi.org/10.1109/TPAMI.2012.213

Kou, F., Ghen, W., Wen, G., \& Li, Z. (2015). Gradient Domain Guided Image Filtering. IEEE Transactions on Image Processing, 24(11). https://doi.org/10.1109/TIP.2015.2468183

Li, Z., Zheng,J., Zhu, Z., Yao, W., \& Wu, S. (2015). Weighted guided image filtering. IEEE Transactions on Image Processing, 24(1), 120-129. https://doi.org/10.1109/TIP.2014.2371234

Li, Z. G., Zhe, J. H., \& Rahardja, S. (2012). Detail-enhanced exposure fusion. IEEE Transactions on Image Processing, 21(1 1). https://doi.org/10.1109/TIP.2012.2207396 
Li, Z.G., Zheng, J., Zhu, Z., \& Wu, S. (2014). Selectively detail enhanced fusion of differently exposed images with moving objects. IEEE Transactions on Image Processing, 23(10). https://doi.org/10.1109/TIP.2014.2349432

Liu, W., Xu, W., Ghen, X., Huang, X., Shen, G., \& Yang, J. (2018). Edge Preserving Piecewise Linear Smoothing using Piecewise Constant Filters. arXiv:1801.06928. https:// arxiv.org/abs/1801.06928

Narasimhan, S. G., \& Nayar, S. K. (2003). Contrast restoration of weather degraded images. IEEE Transactions on Pattern Analysis and Machine Intelligence, 25(6),713- 724. https:// doi.org/10.1109/TPAMI.2003.1201821

Schechner, Y. Y., \& Averbuch, Y. (2007). Regularized image recovery in scattering media. IEEE Transactions on Pattern Analysis and Machine Intelligence, 29(9), 1655-1660. https://doi. org/10.1109/TPAMI.2007.1141

Schettini, R., \& Corchs, S. (2010). Underwater Image Processing: State of the Art of Restoration and Image Enhancement Methods. EURASIP Journal on Advances in Signal Processing, 746052. https://doi.org/10.1155/2010/746052

Tomasi, G., \& Manduchi, R. (1998). Bilateral filtering for gray and color images. In Proceedings of the 1998 IEEE International Conference on Computer Vision, Bombay, India. https:// users.soe.ucsc.edu/ manduchi/Papers/ICGV98.pdf

Torres-Méndez, L. A., \& Dudek, G. (2005). Color Correction of Underwater Images for Aquatic Robot Inspection. In Rangarajan A., Vemuri B., \& Yuille A.L. (eds). Energy Minimization Methods in Computer Vision and Pattern Recognition. EMMCVPR 2005. Lecture Notes in Computer Science, vol. 3757. Springer, Berlin, Heidelberg. https://doi. org/10.1007/11585978_5 